\title{
Doping and Trap States in PPV Light-Emitting Devices
}

\author{
E. Werner, M. Meier, J. Gmeiner, M. Herold, W. Brütting, M. Schwoerer \\ Experimentalphysik II and Bayreuther Institut für Makromolekülforschung (BIMF), \\ Universität Bayreuth, 95440 Bayreuth, Germany
}

The influence of different substrates used for the fabrication of poly-(p-phenylene-vinylene) light-emitting devices on the device characteristics is investigated. In devices prepared on indium-tin oxide substrates doping with $\mathrm{InCl}_{3}$ leads to states with a depth of about $0.15 \mathrm{eV}$ and a concentration of $10^{16}-10^{17} \mathrm{~cm}^{-3}$. This doping is responsible for the observed Schottky diode behaviour in PPV devices on ITO but also leads to considerable photoluminescence quenching. The use of fluorine-doped tin dioxide as anode material causes much lower doping and avoids photoluminescence quenching. An improvement of device properties can be achieved by controlled doping or using partially conjugated PPV.

Doping of conjugated polymers with the aim of replacing metals and the fabrication of organic electronic devices (like e.g. diodes and transistors) was an important research area in the 1980s [1]. The discovery of electroluminescence (EL) in poly-(p-phenylene-vinylene) (PPV) by the Cambridge group in 1990 triggered intense research for optoelectronic devices based on conjugated polymers, combining the charge transport capabilities with optical properties like strong fluorescence in the visible [2]. Due to its good processability and high thermal stability PPV is still one of the most attractive materials for polymer light emitting devices (LEDs) [3].

PPV prepared by the precursor route is particularly subjected to doping reactions since the thermal conversion of the precursor polymer to PPV is performed directly on the anode used for hole injection in LEDs. Especially on an indium-tin oxide (ITO) substrate widely used for the preparation of polymer light emitting devices - oxidation of PPV (leading to doping) can occur through the reaction of indium (or more likely indium oxide) with the $\mathrm{HCl}$ leaving group during the thermal conversion of the precursor to the conjugated PPV. This reaction has been proven by depth profiling of ITO/PPV/Al devices with secondary ion mass spectrometry (SIMS), where enhanced $\mathrm{Cl}$ and In count rates were found throughout the whole PPV layer [4]. With scanning electron microscopy using energy-dispersive $\mathrm{x}$-ray analysis (EDX) the formation of $\mathrm{InCl}_{3}$ has been detected at the ITO/PPV interface [5]. Since $\mathrm{InCl}_{3}$ is known as an oxidizing agent, we believe that this compound is responsible for doping of PPV in the same way as has been shown earlier for $\mathrm{FeCl}_{3}[6]$.

In this paper we will address the influence of different substrate materials on the electrical characteristics of PPV devices. Additionally we will discuss alternative anode materials, controlled doping and modifications of PPV to improve device performance.

In Fig. 1 we compare current-voltage and brightnessvoltage characteristics of PPV devices fabricated on different anode materials (indium-tin oxide (ITO), fluorinedoped tin dioxide (FTO) and gold (Au)). The most striking difference between the three devices is the strong variation of the current in forward direction (Al biased negatively).

In previous

work it has been shown that ITO/PPV/metal devices with low work function metals like $\mathrm{Ca}$ or $\mathrm{Al}$ are well described by a Schottky diode model, where the current is carried predominantly by holes and determined by a Schottky barrier at the PPV/metal interface $[7,8]$. The ITO/PPV contact is regarded as an ohmic contact with negligible injection barrier. Taking into account a serial resistance $R_{B}$ of the PPV bulk, which is considerably larger than in conventional semiconductor Schottky diodes, the I-V characteristics in forward direction can be described well by the modified Shockley equation:

$$
j=j_{S}\left[\exp \left(\frac{q\left(V-I R_{B}\right)}{n k T}\right)-1\right]
$$

where $n$ is the ideality factor and $j_{S}$ the saturation current density.

We have fitted the I-V characteristics of the three devices with the modified Shockley equation (1) between 1 and $5 \mathrm{~V}$ in forward direction. The important parameter in this context is the PPV bulk resistance $R_{B}$ which varies over 5 orders of magnitude between $4 \times 10^{2} \Omega$ for ITO, $1.5 \times 10^{4} \Omega$ for FTO and $7.1 \times 10^{7} \Omega$ for Au. With the device geometry one can calculate the conductivity of the PPV bulk for the respective anode material. One yields values of $4.5 \times 10^{-7}(\Omega \mathrm{cm})^{-1}$ for ITO, $1.2 \times 10^{-8}(\Omega \mathrm{cm})^{-1}$ for FTO and $2.5 \times 10^{-12}(\Omega \mathrm{cm})^{-1}$ for $\mathrm{Au}$. The data for $\mathrm{Au}$ are in good agreement with published d.c. conductivity measurements performed on free-standing PPV films [9]. The drastic change in conductivity from Au to ITO is a clear evidence that the fabrication of PPV devices on ITO substrates leads to doping of the polymer. With FTO as anode material, which is expected to be chemically more inert, the degree of doping must be less compared to ITO.

The concentration and energetic depth of states created by doping has been determined recently using capacitance-voltage spectroscopy and thermally stimulated currents [10]. In ITO/PPV devices the dopant states have energies of about $0.15 \mathrm{eV}$ and a concentration in the range $10^{16}-10^{17} \mathrm{~cm}^{-3}$. With FTO as anode 
the energetic depth is comparable, however, the dopant concentration is lower by a factor of 5 to 10 . For the Au devices we determine the upper limit of the dopant density as $10^{15} \mathrm{~cm}^{-3}$.

The open symbols in Fig. 1 show the electroluminescence light output of the three devices measured simultaneously with the I-V characteristics. For ITO we observe electroluminescence for a bias voltage $V>3 \mathrm{~V}$, the FTO device has an onset voltage of about $7.5 \mathrm{~V}$, and for the Au device no electroluminescence is detectable for voltages up to $25 \mathrm{~V}$ at this thickness. The increase of onset voltage with decreasing doping concentration is due to a transition from a Schottky diode with an inhomogeneous field distribution in the case of ITO to an insulator device in the case of $\mathrm{Au}$, where the applied voltage drops almost homogenously over the whole PPV bulk. In the latter case, the field necessary for electron injection is not reached at the given thickness.

Doping of PPV by the anode material does not only influence the electrical characteristics, in LEDs it can also have important consequences for fluorescence. By investigating photoluminescence spectra of PPV films converted on glass, ITO and FTO we found significant quenching effects in the case of ITO (a reduction by a factor of 20 was observed at the ITO/PPV interface) [10]. With FTO - although we measured considerable doping concentration - no quenching has been observed as compared to glass substrates. Thus FTO can be considered as a new stable anode material for the fabrication of PPV LEDs.

However, as can be seen from Fig. 1, electroluminescence onset voltages in the case of FTO are much higher than with ITO. To overcome this drawback we performed controlled doping of PPV films converted on FTO prior to metal evaporation using solutions of $\mathrm{FeCl}_{3}(0.005 \%$ in acetonitrile) as oxidising agent. As can be seen in Fig. 2 doping reduces the electroluminescence onset voltage of FTO devices by $2 \mathrm{~V}$ and increases current by a factor of 2. However, as the current is still more than one order of magnitude lower than in the case of ITO, quantum efficiency increases by about a factor of 2 . Additionally, light is emitted already at lower currents. Thus the combination of inert anode materials in combination with controlled doping can be regarded as a promising approach to improve device performance.

Another possibility we found to reduce doping of PPV during conversion on ITO is the use of partially conjugated PPV (segmented PPV, s-PPV), where already in the sysnthesis of the precursor polymer H-atoms at the vinylene group are substituted by ester groups, which causes an interuption of the conjugated $\pi$-system at this position after conversion (see inset in Fig. 3). The shorter conjugation length causes a blue shift of absorption and emission spectra (see Fig. 3), from which a decrease of the effective conjugation length from about 10 to 5 monomer units can be estimated. Capacitance-voltage measurements show that the conversion of s-PPV on ITO leads to much lower dopant concentration as compared to con- ventional PPV. I-V characteristics do not display the Schottky-type behaviour, since the current in forward direction is drastically reduced (more then 3 orders of magnitude at $10 \mathrm{~V}$ ) (see Fig. 4). Since the same brightness is achieved at much lower current, quantum efficiencies of devices with s-PPV are higher by a factor of 30 for single layer devices with $\mathrm{Al}$ as cathode and by a factor of 10 for Ca (see Fig. 5). Even in double layer devices with PBD as electron transport layer efficiency can be still increased by a factor of 5 reaching values of $0.3 \%$ (external quantum efficiency). The comparison of PPV and segmented PPV shows that the degree of doping created during device fabrication not only depends on the anode material but also on the chemical structure of PPV (effective conjugation length and perhaps also morphology). This might explain the different device characteristics of PPV devices reported by different groups.

In conclusion, our investigations show that LEDs from precursor PPV are subjected to doping processes caused by the substrate used for device fabrication. The resulting device characteristics strongly depend on the doping concentration in PPV. Doping by $\mathrm{InCl}_{3}$ on ITO substrates is responsible for the formation of a Schottky contact at the PPV/cathode interface, which determines the device characteristics. However this unintentional doping leads to significant photoluminescence quenching. We have found that the use of FTO as stable anode material together with controlled doping of PPV or the use of segmented PPV can lead to significant device improvement.

[1] T. Skotheim, Handbook of Conducting Polymers (M. Dekker, New York, 1986).

[2] J. Burroughes, D.D.C. Bradley, A.R. Brown, R.N. Marks, K. Mackay, R.H. Friend, P.L. Burn, and A.B. Holmes, Nature 347, 539 (1990).

[3] ICSM96 Conference Proceedings, Synth. Met. 84 \& 85, (1997).

[4] G. Sauer, M. Kilo, M. Hund, A. Wokaun, S. Karg, M. Meier, W. Rieß, M. Schwoerer, H. Suzuki, J. Simmerer, H. Meyer, and D. Haarer, Fresenius J. Analyt. Chem. 353, 642 (1996).

[5] M. Herold, J. Gmeiner, C. Drummer, and M. Schwoerer, J. Mater. Sci. at press (1997).

[6] R. Mertens, P. Nagels, R. Callaerts, M. Van Roy, J. Briers, and H.J. Geise, Synth. Met. 51, 55 (1992).

[7] W. Rieß, in Organic Electroluminescent Materials and Devices, edited by S. Miyata and H. Nalwa (Gordon\&Breach Science Publishers, London, 1996).

[8] S. Karg, M. Meier, and W. Rieß, J. Appl. Phys. at press (1997).

[9] J. Gmeiner, S. Karg, M. Meier, W. Rieß, P. Strohriegl, and M. Schwoerer, Acta Polym. 4, 201 (1993).

[10] W. Brütting, M. Meier, M. Herold, S. Karg, and M. Schwoerer, submitted to Chem. Phys. (1997). 


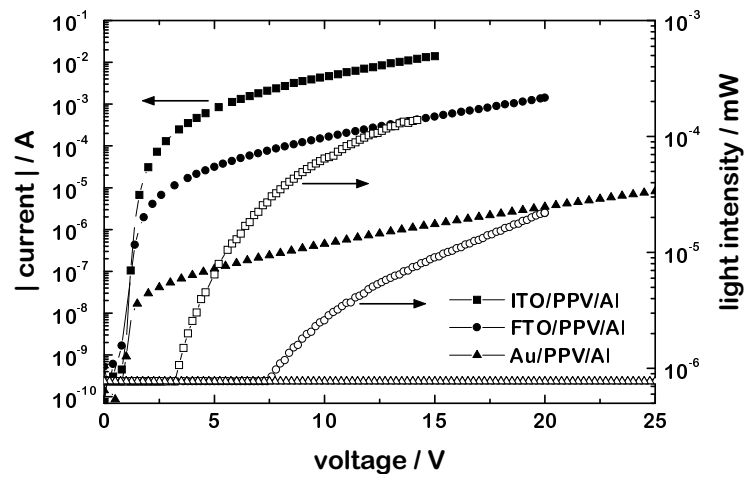

FIG. 1. I-V characteristics and light output of PPV devices fabricated on different substrate materials. The PPV thickness of all devices was about $450 \mathrm{~nm}$ and the active device area $0.25 \mathrm{~cm}^{2}$. The flat line with a light intensity of $8 \times 10^{-7} \mathrm{~mW}$ is the detection limit of our setup.

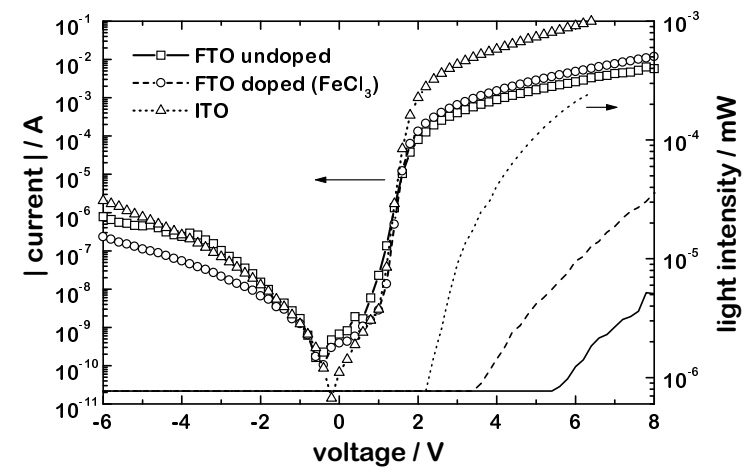

FIG. 2. I-V characteristics and light output of PPV devices fabricated on ITO and FTO (undoped and doped with $\mathrm{FeCl}_{3}$ ). The PPV thickness of all devices was about $200 \mathrm{~nm}$.

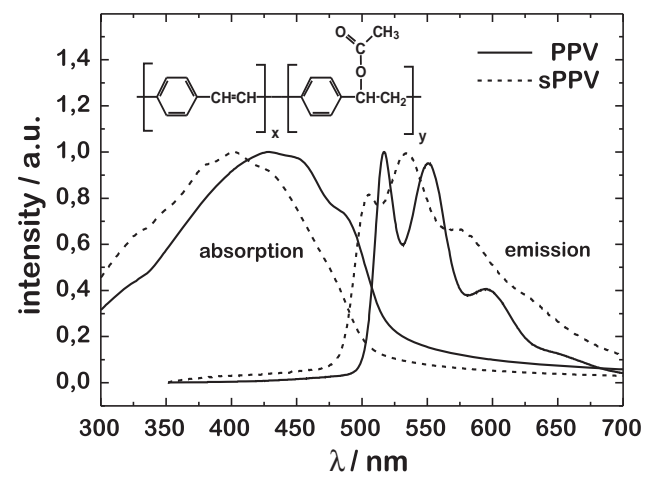

FIG. 3. Absorption and emission spectra of PPV and a partially conjugated modification of PPV (s-PPV), whose chemical structure is also shown.

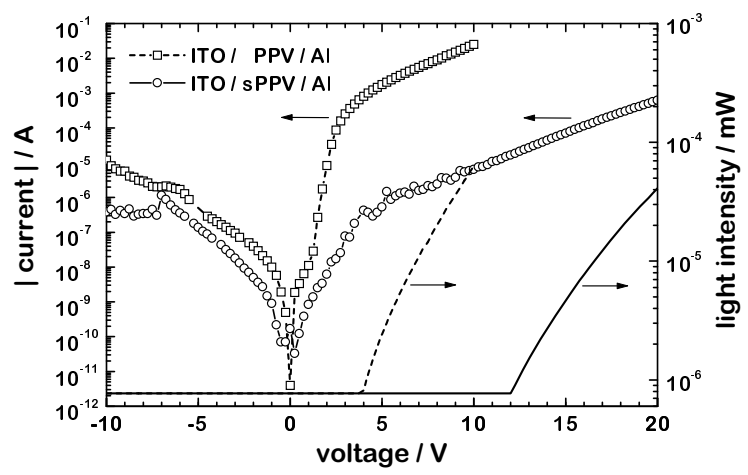

FIG. 4. I-V characteristics of devices with PPV and segmented PPV as active layer.

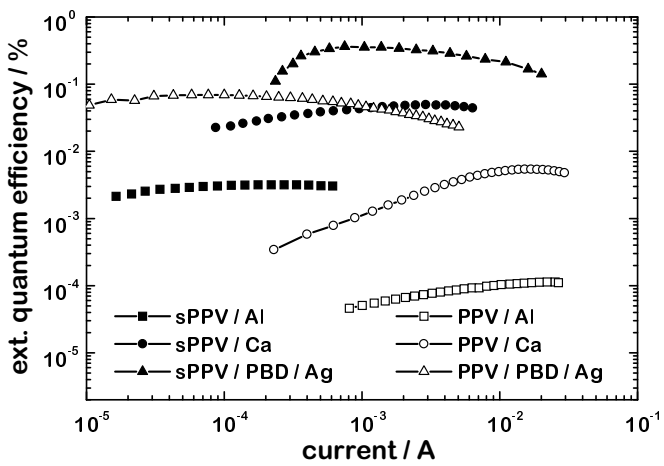

FIG. 5. External quantum efficiencies of devices with PPV or segmented PPV. All devices have ITO as anode material. 